\title{
Brief Intervention to prevent HIV, STI and unintended pregnancies: preliminary results of a feasibility study from the perspective of healthcare providers in Peru
}

Jean Pierre Jiron ( $\nabla$ jean.jiron@upch.pe)

Center for Interdisciplinary Studies in Sexuality, AIDS and Society, Universidad Peruana Cayetano Heredia

\section{Clara Sandoval}

Center for Interdisciplinary Studies in Sexuality, AIDS and Society, Universidad Peruana Cayetano Heredia

\section{Juan Carlos Enciso}

Center for Interdisciplinary Studies in Sexuality, AIDS and Society, Universidad Peruana Cayetano Heredia

\section{Ana Sofía Vasconcelos}

UNDP-UNFPA-UNICEF-WHO-World Bank Special Programme of Research, Development and Research Training in Human Reproduction (HRP), Department of Sexual and Reproductive Health and Research, WHO

\section{Karel Blondeel}

UNDP-UNFPA-UNICEF-WHO-World Bank Special Programme of Research, Development and Research Training in Human Reproduction (HRP), Department of Sexual and Reproductive Health and Research, WHO

\section{Nataliia Bakunina}

Institute for Leadership and Health Management, I.M. Sechenov First Moscow State Medical University, Sechenov University

\section{Galina Lesco}

National Resource Centre in Youth Friendly Health Services NEOVITA

\section{Igor Toskin}

UNDP-UNFPA-UNICEF-WHO-World Bank Special Programme of Research, Development and Research Training in Human Reproduction (HRP), Department of Sexual and Reproductive Health and Research, WHO

\section{Rob Stephenson}

Department of Systems, Population and Leadership, School of Nursing, University of Michigan

\section{Carlos F. Caceres}


Center for Interdisciplinary Studies in Sexuality, AIDS and Society, Universidad Peruana Cayetano Heredia

\section{Research Article}

Keywords: Healthcare providers, Brief sexuality-related communication, sexual health, Behavior change techniques

Posted Date: July 1st, 2021

DOl: https://doi.org/10.21203/rs.3.rs-601818/v1

License: (c) (7) This work is licensed under a Creative Commons Attribution 4.0 International License. Read Full License

Version of Record: A version of this preprint was published at BMC Health Services Research on November 12th, 2021. See the published version at https://doi.org/10.1186/s12913-021-07229-y. 


\section{Abstract}

\section{Background}

Brief interventions have proven to be valuable instruments for the treatment and care of clients with diverse health needs, due to their potential to impact both the individual and the population. In this regard, the Brief Sexuality-Related Communication (BSC) is presented as a viable and effective alternative for addressing sexual and reproductive health problems, assessing risk behaviors and motivating clients to generate behavioral change. Since health providers are key actors in treatment and prevention, it is essential to know their perceptions about the BSC intervention, as well as its acceptability in different contexts, with diverse client populations. Thus, the following paper reflects the findings of the perceptions and experiences of health providers in Peru from the first phase of the Feasibility study of a BSC intervention to prevent STIs and unintended pregnancies.

\section{Methods}

This is the first phase of a multisite and multiphase study of the feasibility of a BSC intervention. We conducted twenty in-depth interviews (IDI) with health care providers (physicians, obstetricians, psychologists, nurses and peer counselors) recruited from three health care institutions in Peru: The Tahuantinsuyo Bajo Maternal and Child Center (CMI) and the San José Maternal and Child Center, both located in the capital city, Lima; and La Caleta Hospital located in Chimbote, northern coast of Peru. Participating health providers included those working at the HIV/STI Reference service and the family planning/reproductive health service. The IDI addressed three domains: 1) Acceptability of the BSC intervention; 2) Perceived willingness to implement the BSC intervention; and 3) Considerations for the Implementation of the BSC intervention.

\section{Results}

Health providers expressed high acceptance of the BSC intervention, considering it as a useful and effective instrument to address sexual and reproductive health problems with all clients; however, some providers had some concerns about the real impact of the intervention to achieve significant behavior change. On the other hand, health providers showed high willingness to learn and implement the BSC intervention, affirming their commitment to learn new techniques and strategies that could allow them to improve their knowledge and the quality of their care. Health care providers consider it necessary to take into account the barriers that arise in the implementation of the BSC intervention, such as the structural limitations to access, the providers' abilities to deliver the intervention effectively, and the participants' reception of the intervention. Finally, providers consider it essential to establish the BSC intervention in a normative framework that allows it to receive the support of the health departments and eventually enforces implementation. 


\section{Conclusions}

Health providers consider the BSC intervention as an interesting and exciting behavioral intervention to deal with the sexual and reproductive health issues existing in different populations, and seemed highly willing to adapt and implement it, hoping that it become beneficial to all client populations to prevent HIV/STIs and unintended pregnancies.

\section{Introduction}

It is essential for all people to achieve a state of comprehensive sexual health and well-being, 1, 2, 3 and whether people can achieve this will largely depend on their access to comprehensive information about sexuality, their vulnerability to the adverse consequences of their sexual behavior, and access to highquality sexual health care, as well as an environment that helps them affirm and promote their sexual health. ${ }^{4,5}$

In this regard, brief interventions are considered as informal counseling and information on certain types of harms and risks associated with risky behaviors, and aim to engage certain populations who are not yet ready for change. These brief interventions have become increasingly valuable for the treatment and care of clients, because of their low-cost benefits and proven effectiveness to handle a great diversity of public health issues. 6,8 Due to their potential to impact both the individual and the population, helping individuals to understand risk behaviors and motivating them to generate significant changes in their health care, brief interventions have been well received by health care providers and policy makers who consider them effective tools for prevention of risk behaviors in different public health arenas. ${ }^{9}$ While brief interventions are based on a large scope of theoretical streams, the use of Motivation Interviewing principles ${ }^{10}$ is being more and more widely adopted to deliver brief interventions addressing health issues and achieving behavior change. These brief interventions share general principles like: "Feedback on existing risk situations and potential/real damages; the responsibility for the change rests with the person/patient; advice on practical strategies to reduce risky behaviors; a menu of options to help achieve behavior change; empathic delivery; and development of self-efficacy by healthcare providers". 9

In that sense, the Brief Sexuality-related Communication (BSC) is defined in the WHO's guidelines ${ }^{11}$ as an opportunistic, dynamic communication process between a patient and a trained health care provider that includes the addressing of diverse problems and difficulties related to sexuality and the promotion of sexual wellbeing, taking into account biological, psychological and social dimensions. ${ }^{12}$ The BSC aims to identify current and potential sexual issues and motivate those at risk to change their sexual behavior or maintain safe sexual behavior. $6,12,13$ In addition, the BSC helps clients to re-evaluate their emotions and behavior for a better understanding of their sexual health, promoting critical reflection and setting clear goals, developing their capacity for self-regulation, and improving their autonomy and satisfaction. ${ }^{11,12}$ The BSC is based on the theoretical dimensions of the "information, motivation and behavior" model, ${ }^{14,15}$ 
and uses Motivational Interviewing (MI) techniques ${ }^{6}$ with a "client-centred" approach, ${ }^{11}$ which respects clients' ideas, feelings, expectations and values. ${ }^{13}$

Healthcare providers are in a unique position to identify and intervene clients in risky situations and offer them a variety of options to address their sexual health issues. ${ }^{8}$ In that sense, clients often have confidence on healthcare providers' expertise to address their concerns, and the information provided by healthcare providers plays a crucial role not only in the treatment, but also in the prevention of sexual health issues. In this way, health providers are the main actors in the delivery of health services, and it is very important that they are able to build a bond of trust and respect with clients that facilitates health care. ${ }^{16,17}$ At the same time, it is necessary for health providers to stay informed and trained in order to deal with the various issues that arise in sexual health. $18,19,20$

To be able to offer effective, evidence-based sexual health care, providers should remain up-to-date concerning methods and techniques useful for the promotion of sexual health and prevention of related problems. When these conditions are not met, health providers can feel that their abilities are not adequate to screen and counsel clients with sexual health issues. ${ }^{8,11}$ That may lead to discomfort and insecurity when talking about sexual practices, particularly with populations with special sexual health needs. ${ }^{17,19}$ Therefore, training opportunities that cover an update on technical topics and that complement their intervention's skills are often welcomed by health providers.

Although the effectiveness of brief behavioral interventions for the prevention of STIs/HIV has been shown ${ }^{21}$, it is relevant to assess the feasibility of the BSC intervention in public facilities in lower-middle income countries, particularly when working with clients belonging to diverse vulnerable and stigmatized populations in various sociocultural contexts. ${ }^{4,11,12}$, In this way, the BSC intervention generates new opportunities for health care providers to help their clients change behaviors that are relevant to their sexual health care. ${ }^{13,22}$

This report comes from the first phase of a feasibility study of the brief sexuality-related communication (BSC) intervention in Peru, as part of a WHO multiphase and multisite study ${ }^{22}$, in which qualitative information was evaluated on the perceptions and attitudes of healthcare providers about the BSC intervention, and whether it would work in their daily practice offering sexual health services. ${ }^{13}$

\section{Study Setting}

Peru is described as having an HIV epidemic concentrated on men who have sex with men (MSM), and transgender women (TW ${ }^{23-24}$. The estimated number of people over 15 years of age living with HIV in 2012 was $72,000 .{ }^{25}$ It has been estimated that $84 \%$ of new cases and $55 \%$ of prevalent cases occur in key populations. ${ }^{26}$ HIV prevalence among MSM varies between 10 and $20 \% 24,27$; and among TW it can reach $30 \% 28$, with the very high risks for acquiring HIV in that group linked to high rates of sex work and drug use, themselves linked to extreme marginalization, social exclusion and violence. In Peru, 
homophobia and transphobia are still prevalent, and situations of discrimination and exclusion experienced by the lesbian, gay, bisexual, transgender and intersex (LGBTI) community are common in everyday life.

Likewise, in Peru the adolescent birth rate is estimated at 53 births per 1,000 adolescents between the ages of 15 and 19 (2017-2018), and 12.6\% of adolescents were mothers or became pregnant for the first time while in that age group ${ }^{29}$. Among pregnant adolescents, $64.2 \%$ did not seek or want their pregnancy at that time during $2019 .{ }^{29}$ Hence, the adolescent pregnancy rate has hardly changed in more than 20 years and, in relation to the national goal of reducing adolescent pregnancy by $20 \%$ in 2021 , no progress has been made. ${ }^{30}$ Adolescent motherhood continues to be the harshest expression of social injustice, poverty and vulnerability of women who come from rural areas, who are indigenous or come from the Amazon, and who struggle to have access to education and health, while live in a situation of poverty and exclusion. ${ }^{30,31}$

In response to these sexual and reproductive issues, the Peruvian state has been implementing the Technical Health Standard for Comprehensive Adolescent healthcare, ${ }^{32}$ which includes universal access to adolescent health and unrestricted medical care, covering a specialized package that includes sexual and reproductive health, physical, nutritional and mental health services. And specifically speaking about the prevention of child/adolescent pregnancy, the Technical Standard for Family Planning ${ }^{33}$ allows adolescents to access SRH services and contraceptive methods autonomously, without parental authorization. However, some of the health providers still limit adolescents' access to contraceptive methods. ${ }^{30}$ Well-trained and sensitized healthcare providers with a human rights approach are needed to address adolescents' diverse sexual and reproductive health needs.

\section{Methods}

This is part of a multisite and multiphase study of feasibility of a BSC intervention in primary health care settings across four phases proposed by WHO. These findings are part of Phase I of the study which involves the collection of formative, qualitative data to examine health care providers' perceptions of the feasibility of the intervention.

We used a phenomenological approach to explore the perceptions and attitudes of health care providers towards the BSC intervention in the SRH services (HIV, sexually transmitted infections [STI], pre-natal care, family planning, etc.), that work with MSM, TW, female sex workers (FSW), people living with HIV, adult women and adolescent females.

Twenty IDI were conducted between September 2018 and March 2019, with health care providers (physicians, midwives, nurses, psychologists and peer counselors) recruited from three health care facilities. Two of those facilities are maternal and child centers (CMI) in Lima with a status of primary care facility: the CMI Tahuantinsuyo Bajo, located north of Lima, and the CMI San José, located south of Lima. The third one is the La Caleta Hospital in the Ancash Region, located in the northern coast of Peru. 
All these facilities have HIV/STI referral centers (CERITS), where all clients are seen for the prevention and treatment of HIV \& STIs, and also offer reproductive health services to adolescent and adult women (i.e. contraceptive methods, prenatal care and other).

Before initiating the IDI, the BSC intervention was described to health providers. The IDI addressed health care providers' perceptions about the BSC intervention and explored providers' knowledge to address clients' specific needs in sexual health; sensitive issues to address with client populations; comfort working with key populations and discussing sexual health issues; strategies for addressing risk and prevention issues; acceptability of the intervention; barriers to delivering the intervention; and concerns about the intervention. These aspects were structured on three domains: 1) Acceptability of the BSC intervention, which seeks to explore the attitudes that health providers have towards the intervention, its perceived utility, and concerns and doubts about its impact; 2) Perceived willingness to implement the BSC intervention, which explores their willingness to participate in the training to learn the intervention, what aspects need to be reinforced during the training and their willingness to deliver the intervention; and 3) Considerations for the Implementation of the BSC intervention, addressing intervention feasibility, necessary conditions for its sustainability, barriers and difficulties for its implementation, and cultural adaptation of the intervention into the already-existing client-provider appointment were explored in interviews.

Every IDI lasted approximately 45-60 minutes, was audio-recorded and subsequently transcribed for analysis. Each transcript was reviewed for accuracy and any identifying information was removed. The IDIs were conducted by members of the research team in Peru. A code book was developed that helped structure the information recollected. During the coding process, several new codes and relational themes were identified, that allowed the creation of categories that were discussed and restructured by the research team members. We used Dedoose 8.3.35 software for the coding and analysis of interview transcripts, according to relational, familiarity and saturation criteria, applying an analytical framework. Several meetings were necessary to discuss and restructure the analysis based on the findings and interpretations that later became key study findings.

\section{Results}

\section{ACCEPTABILITY OF THE BSC INTERVENTION}

\section{Provider attitudes towards the BSC Intervention}

Health care providers consider the BSC intervention to be a potentially effective tool that can contribute to the orientation and counseling of clients, especially with adolescents in search of a space where they can get answers about their sexuality and SRH without fear of being judged. Additionally, it would be a very useful tool to face the current landscape in sexual and reproductive health in the country.

"It would be excellent because it is always important to handle this situation with young people who are quite disoriented; apart from the fact that they lack privacy, they have nobody to approach for help; they 
are afraid to ask parents, who also feel insecure to talk to them about these issues. So, who better than health professionals to offer them the confidence they need and help them with these issues? And to prevent pregnancy among adolescents, (a problem) that never ceases to grow". (Interview 6, Female)

As a manner of self-criticism, providers manifest that despite having previously worked with different person-centered behavioral change techniques, they have not seen significant changes in the incidence of HIV and STI cases. Therefore, providers feel the urgent need to work with new strategies and techniques aimed at behavior change that are effective in the prevention of HIV and STIs.

"Of course, because by focusing on the person we can change behaviors; because so many strategies have been implemented, so many activities to prevent STIs and HIV... and the statistics continue to show new cases, then something happens and we can't change anything fundamental, because the most difficult thing in someone is to achieve behavioral change". (Interview 5, Female)

\section{Perceived Utility of the BSC Intervention}

Health care providers consider that it would be very useful to address directly with clients the possibility of improving their sexual and reproductive health care by using innovative strategies. Providers also consider that clients in general would greatly benefit from this intervention because they would perceive the empathy and interest from providers to help them solve their sexual health issues and improve their wellbeing.

"Yes, I think so, because they are going to feel that they care about you, and that you want to do something for him or her and that you really listen to them. People come here because they want to be heard". (Interview 18, Male)

"Of course, because when you open it up, the client talks to you about other topics that are also suddenly generating anxiety at that moment and they are a little more liberated from suddenly believing that what they were thinking was something strange and not. Talking about sexuality is part of ourselves and we are also a consequence of our sexuality". (Interview 3, Female)

In this way, health care providers consider that the BSC intervention will be of great help to deal more fully with risky situations. Furthermore, they state that the intervention addresses comprehensive care, including other personal and emotional aspects. This would reinforce prevention work at a deeper and more complex level.

"...It will help us greatly (to promote) changes in our patients' sexual behaviors, which is what we also seek with our counseling, to change or help prevent risk behaviors more than anything, offering them the knowledge and helping them avoid risky behaviors". (Interview 13, Female)

"It would be like doing prevention work in many aspects, because one thing they come here for is to rule out if they have any STIs. So, it would be to prevent with comprehensive information, because not only are we going to give them information about STIs, but we are also going to talk about the personal part, 
about controlling your drive, your emotions, and about your self-esteem, so that then they use protection and can take care. So that would be preventive, it seems very good to me". (Interview 20, Female)

\section{Concerns and doubts about BSC Intervention's impact}

However, health care providers stated that, depending on the capabilities of who delivers the intervention and how it is delivered, it will have a positive impact on the clients, helping them to recognize strengths and weaknesses and to generate concrete behavioral changes (to improve) their sexual health.

"I think it would be very useful, because depending on the counseling, they will apply it in their lives, and that will help them to know what strengths they have that they did not even know about, or that they had not even perceived they had. And weaknesses that they were not conscious about either". (Interview 18, Male)

On the other hand, health providers expressed various doubts and concerns about the real impact the intervention can make to tackle sexual health issues. They comment on clients who re-engage in the same risk behaviors, stating that a single session of the intervention can't be sufficient to generate significant behavioral change, because it is a long and constant process in which the health provider accompanies the client. In addition, providers manifest that clients' final decisions regarding their sexual health issues are also influenced by their environment, which is not under the providers' control.

"Well, it is assumed that, if we are going to take some time to work on a single topic, it is because we have to do it, and (that decision) will be effective to (ensure we) achieve something little by little, to make a change, because changes among people do not happen overnight, and because we can say one thing, but when we go home, father, mother and the whole environment may influence our decisions (to do otherwise)". (Interview 14, Female)

"In the case of patients who have an STI, we also try to follow the same procedure a bit, but we must insist on why they had an STI, and what can happen if they continue with the same behaviors. Obviously what counseling is looking for is a change in behavior, but we know that it will not be achieved with counseling only or in a few months. Rather it is a whole process that takes some long time". (Interview 15, Female)

\section{PERCEIVED WILLINGNESS TO IMPLEMENT THE BSC INTERVENTION}

\section{Willingness to perform the BSC Intervention}

Health care providers expressed their willingness to implement the BSC intervention by saying that it can benefit both clients and themselves as long as the methodology to be implemented meets the criteria of confidentiality, privacy and respect while being focused on the welfare of the client.

"... If the intervention continues to meet the criteria of confidentiality, privacy, then, (if) used by health staffing search of the same results, it will be beneficial for the health provider and the patient". (Interview 


\section{5, Female)}

Similarly, healthcare providers remain open to learning new methods and techniques that can help them address sexual health issues with their clients, and they are happy to receive this support from various health institutions.

"If there are changes for the good of the population, welcome. We are happy to support them, because now they are with the WHO, but the idea would be for the Ministry of Health to take it, and if they are also going to support us with some logistics, in good time. We should not say no to the changes that are coming". (Interview 14, Female)

\section{Willingness to participate in the training for the BSC Intervention}

Likewise, health providers consider it necessary and essential that they are able to carefully review the BSC intervention in depth. Therefore, the training is crucial so that the team can learn, evaluate and adapt the strategy in depth. In addition, health providers report that it would be very useful for them to learn, as well as to reinforce the topic of social skills.

"Well, any project or program that you want to logically implement, you have to first receive the training to implement it. (Then you) present it as a group in a technical meeting, with the whole team, and I think the whole team would also agree. With anything to improve the consultation and patient care I think there would be no problem". (Interview 13, Female)

"First is the issue of what the BSC intervention is (or consists of). Everything that corresponds to the technical part, strengthening the skills issue as well, in the search for information about the different populations that we deal with, it seems important to me, how to reach that client who is different". (Interview 5, Female)

\section{Necessary aspects to reinforce in the training of the BSC Intervention}

For the training sessions, health providers suggest that concepts related to gender, gender identity, and other issues that allow them to address the specific needs of each of the client populations should be reinforced.

"There should be much more in itself, addressing gender issues, gender identity, so that we have much more to talk about when intervening the population, we manage". (Interview 10, Female)

Similarly, they consider it vital to reinforce the strategies for approaching issues of sexual rights, the use of contraceptive methods, the resolution of partner conflicts and especially the issue of violence, since these are the cases they frequently deal with.

"Sexual rights, especially the issue of violence, the issue of sexual rights, the issue of the couple relationship, the issue of contraceptive methods". (Interview 8, Female) 


\section{CONSIDERATIONS TO IMPLEMENT THE BSC INTERVENTION}

\section{Feasibility of the BSC Intervention}

Health care providers stated that human and material resources as well as training of the team of health professionals are vital for intervention implementation. Taken together it will play a crucial role in the feasibility of the BSC intervention.

"Well, I believe that as long as there are human resources and the necessary materials to implement it and maintain it, then we will all be able to do it". (Interview 13, Female)

In order to perform the intervention in the best possible way, the interactions between health care providers and clients must meet certain conditions by which the clients feel comfortable, safe and confident enough to address their sexual health issues. It will depend on the provider's skills to apply the principles of the intervention and their capacity to reach clients with empathy, compassion and respect.

"It all depends on who speaks to them... rather, the people who are going to give this type of intervention must be suitable people, who have that freedom, who know how to gain empathy, who know how to reach the patient, who have those qualities". (Interview 4, Female)

"Many times they shy away from telling you many things, or you do not give them confidence because of the tone of voice, or sometimes how you ask, because they may think that you are judging them". (Interview 7, Female)

\section{Conditions for the Sustainability of the BSC Intervention}

Providers state that for the intervention to be sustainable, it is necessary to build communication networks between health services and those responsible for making decisions in health facilities. It is vital that a commitment is maintained by decision makers to guarantee the supply of necessary material conditions, as well as the availability of staff to carry out the intervention in health services.

"(We) try to get others involved... one of the things we do in this HIV and STI REFERENCE CENTER (CERITS), despite the fact that we do not have an assigned population, and that we are not a health network, is to share the information we have, all the time. We are personally committed to transfer to other health networks any training we receive". (Interview 5, Female)

"That also depends on the head; it is a bit complicated... It depends on the Director, because sometimes a director comes up with a new idea, the other director says no, and many colleagues abide by that". (Interview 9, Female)

\section{Barriers and difficulties to implement the BSC Intervention}

However, there are some structural barriers that represent difficulties in care, related to reduced spaces and inadequate infrastructure of the health facility, which hinder confidentiality; inflexible schedules, and 
staff shortages, which generate long waiting times for clients.

"We don't have the ideal facility; in other words, space is a limitation ... another is the time we have to offer counseling". (Interview 7, Female)

"Barriers include time - because patients do not have much time; and also a waiting list; logically that will cause discomfort. Another potential barrier is the rejection of patients, who suddenly do not want to receive the necessary guidance or who are in a hurry, basically that". (Interview 13, Female)

\section{Providers' perceived self-efficacy to implement the BSC Intervention}

Health providers state that, depending on their capabilities, it will take time for them to tailor the BSC intervention to their regular work, but stated that they are willing to learn the intervention to benefit their clients and address their sexual health issues in the best possible way.

"Maybe at the beginning yes, more than anything on the part of the health personnel, as in everything, it is a process, and for the health personnel also if it is a new methodology, a new strategy, it will take a little bit of time to adapt, but if it is so beneficial and effective, it is okay to implement it". (Interview 15, Female)

Time can be a factor that hinders intervention, as clients often prefer short visits. While there are clients for whom longer visits would not represent a problem, health providers still recommend to keep visits as short as possible.

"Sure, as long as it doesn't last long. Because, when the visit takes much longer, some clients may feel uncomfortable..., but it is very diverse... on the one hand there are patients who want to listen to you and want to continue talking; on the other hand, there are those who come in a hurry and have to go to work immediately. That really depends on each patient". (Interview 13, Female)

\section{Discussion}

Our findings about perceptions of the BSC intervention among health care providers in Peru have shown that the BSC intervention has been perceived as a potentially effective instrument for sexual and reproductive health care, aiming to assist the clients to reflect on the reasons for their risk behaviors, reevaluate their emotions and understand their sexual health better, motivating them to change their behaviors. ${ }^{11}$ In addition, health care providers highlight the potential benefit of improving their ability to establish an empathic interaction with clients, helping them set goals and develop their autonomy, while simultaneously the BSC intervention gives the client greater satisfaction and security to face their sexual health concerns. ${ }^{12}$ Moreover, due to its innovative nature, the BSC intervention is seen by health providers as a groundbreaking opportunity to address various sexual and reproductive health problems in different populations, linked to the high rates of unintended pregnancies in recent years, 29,30 and the multi-layered vulnerability faced by key populations regarding HIV and STIs. ${ }^{24,28,31}$ It is important to clarify, however, 
that our findings here represent providers' perceptions about BSC after getting explanations of the principles of BSC, but before being trained on and directly exposed to this intervention.

From an instrumental viewpoint, it is considered essential for health care providers to remain up-to-date with new interventions and techniques aimed at changing behaviors for HIV/STI prevention. ${ }^{18,19,20}$ In fact, health care providers consider that being trained to implement the BSC intervention will allow them to address different needs in sexual and reproductive health, in line with findings in other studies. ${ }^{6,11}$

Although the BSC intervention concept is naturally appealing to health care providers due to the flexibility it implies to approach sexual health issues, providers also express some doubts about the real impact it may have on clients' behaviors. Some providers expressed that addressing sexual health issues is part of a much larger and complex process that can hardly be addressed in a single session; or added that the various situations of inequality and vulnerability in which many clients find themselves must be considered. While the BSC intervention has been designed to not only help patients make and enact behavioral change decisions, but also to make them feel more in control of their health through positive change ${ }^{11,12,13}$, it is true that external factors play a key role, and they should be addressed during the training.

Regarding their willingness to learn and implement the intervention, health care providers expressed their commitment to learn in detail the guidelines of the BSC intervention and adapt it to their medical consultations, since they consider that strengthening their skills, would be very useful for them at a professional level, as well as for clients, offering them much more effective alternatives to face their sexual health issues. While we feel this enthusiasm is positive, we also realize that providers normally welcome any training that offers to help them innovate and improve their practice, ${ }^{16,18,20,21}$ and have to observe how this translates into actual commitment to change in real life.

Health care providers also state that it is necessary to address strategies to approach vulnerable populations in the BSC intervention trainings, and that they would like to learn much more about aspects such as gender identity, human rights, intimate partner violence, sexual abuse, abortion, among others, since those are some of the sexual and reproductive health problems most frequently encountered when seeing clients. This reflects a perspective expressed by providers across several studies in Peru. ${ }^{28,30,31}$

Regarding the necessary considerations to be able to effectively implement the BSC intervention, health care providers stated that it is essential to have the logistical resources that allow them to deliver the intervention, including appropriate training. Moreover, providers emphasize that, in order to ensure the sustainability of this intervention, it must be incorporated into a national regulation that provides legitimacy and turns it into a regular tool to be used in sexual and reproductive health services. ${ }^{32,33}$

Clearly, there are various structural barriers within health facilities that represent a challenge for the implementation of this intervention, including the health facility infrastructure, inflexible schedules, lack of health personnel and limited times to offer the intervention. Some providers indicate that their capacity 
to adapt and execute the intervention in their regular work with clients will be tested. ${ }^{19,20}$ While most of them have expressed a high desire to implement the intervention, they acknowledge that it does not ensure implementation with a high degree of fidelity, which can affect effectiveness. This emphasizes the need for a specific policy that is enforced in order to make the implementation of the BSC intervention possible.

Finally, it is crucial for health providers to consider the variability of clients' reactions to the offer of an indepth, more interactive intervention. While some might welcome it, others might be bothered by the extra time it demands, or find it intrusive and unwarranted. ${ }^{17,} 19$ Such feedback is important for any effort to adapt the BSC intervention to the specific needs of the populations served by public HIV/STI and SRH services in Peru, and possibly in many parts of Latin America.

\section{Conclusions}

Healthcare providers express high acceptance of the BSC intervention as a potentially effective instrument to address sexual and reproductive issues with diverse client populations. These perceptions about the BSC intervention were expressed after it was described to them, but before they had been trained and exposed to it. Many health providers indicated they were willing to implement the BSC intervention, and felt very motivated to receive the intervention training, considering that it could become very helpful to improve their skills and achieve better results when addressing their clients' sexual health concerns. However, some expressed skepticism about its capacity to elicit significant behavioral changes and lead to improved clients' sexual health given its brevity. It is also necessary to consider the various aspects that can hinder the implementation process of the BSC intervention, including the structural conditions of health facilities, the ability of health providers to deliver the intervention with fidelity, and some clients' reaction to the offer of a more in-depth, more comprehensive interaction that they find too lengthy or simply unwarranted. In conclusion, these findings portray a generally positive disposition among providers, in spite of some challenges, and allow us to proceed to subsequent phases of this study.

\section{Declarations}

\section{Ethics approval and consent to participate}

This study has the ethical approval of the WHO Ethics Review Committee and the Institutional Ethics Committee of the UPCH. Ethical approval considers compliance with University standards, scientific and ethical guidelines, the risk / benefit balance, the qualification of the research team and the confidentiality of the data, carried out in accordance with all established guidelines and regulations.

Before proceeding with data collection, all study participants were duly informed about the objective of the study, the implications of their participation, its benefits, and their rights as participants. All this was 
detailed through the review of an informed consent that the subjects signed voluntarily, agreeing to participate in the study.

\section{Consent for publication}

NA.

\section{Availability of data and materials}

The data sets generated and analyzed during the current study are not publicly available because due to their qualitative nature, they were processed to draw conclusions from unstructured and heterogeneous data that are not expressed in a numerical or quantifiable way; but are available from the corresponding author on reasonable request.

\section{Competing interests}

The authors have declared that no competing interests exist.

\section{Funding}

The authors received no specific funding for this work.

\section{Authors' contributions}

JPJ wrote the first draft of the manuscript and incorporated the changes suggested by other authors. CS supervised the qualitative data analysis and provided comments to the first manuscript and JCE assisted with the qualitative data collection. RS, IT, and ASV provided substantial suggestions for two versions of the manuscript, and NB, GL, and KB provided additional comments on the latest version of the manuscript. CFC guided the process and reviewed three versions of the manuscript.

\section{Acknowledgements}

On behalf of all the authors of this study, we want to thank mainly the health care providers from the different sites in Peru who participated in the project. This work would not have been possible without their help, and we are entirely grateful for the dedication they give to their patients and for the commitment they have to continue creating change in public health.

\section{References}

1. World Health Organization. Defining sexual health: report of a technical consultation on sexual health. Geneva: World Health Organization; 2006.

2. World Health Organization. Sexual and reproductive health of women living with HIV/AIDS: Guidelines on care, treatment and support for women living with HIV/AIDS and their children in resource-constrained settings. Geneva: World Health Organization; 2006. 
3. World Health Organization. Developing sexual health programmes: A framework for action. Switzerland: World Health Organization; 2010.

4. Cooper B, Toskin I, Kulier R, Allen T, \& Hawkes S. Brief sexuality communication-a behavioral intervention to advance sexually transmitted infection/HIV prevention: a systematic review. Royal College of Obstetricians and Gynecologists; 2014; 92-103.

5. Hawkes S. Sexual health: a post-2015 palimpsest in global health? The Lancet; 2014; $2 ; 377-378$.

6. Dunn C, Deroo L., \& Rivara FP. The use of brief interventions adapted from motivational interviewing across behavioral domains: a systematic review. Addiction; 2001; 96; 12; 1725-1742.

7. Vasilaki El, Hosier SG, \& Cox WM. The efficacy of Motivational Interviewing as a brief intervention for excessive drinking: A meta-analytic review. Alcohol and Alcoholism; 2006; 41; 328-335.

8. World Health Organization. Brief intervention for hazardous and harmful drinking: a manual for use in primary care. World Health Organization; 2001.

9. Beyer F, Lynch E, Kaner E. Brief interventions in primary care: An evidence overview of practitioner and digital intervention programmes. Current Addiction Reports; 2018; 5; 265-273.

10. Heather N. Interpreting the evidence on brief interventions for excessive drinkers: The need for caution. Alcohol and Alcoholism; 1995; 30; 287-296.

11. World Health Organization. Brief Sexuality-related Communication: Recommendations for a public health approach. Geneva: World Health Organization; 2015.

12. Toskin I, Cooper B, Troussier T, Klugman B, Kulier R, Chandra-Mouli V, \& Temmerman M. WHO guideline for brief sexuality-related communication: implications for STI/HIV policy and practice. Reproductive Health Matters; 2015; 23; 177-184.

13. Toskin I, Bakunina N, Gerbase A, Blondeel K, Stephenson R, Baggaley R, Mirandola M, Aral S, Laga M; Holmes K, Winkelmann C. A combination approach of behavioral and biomedical interventions for prevention of sexually transmitted infections. Bull World Health Organ, 2020; 98; 431-434.

14. Fisher W, Fisher JD, \& Harman J. The Information-Motivation-Behavioral Skills Model: A General Social Psychological Approach to Understanding and Promoting Health Behavior. Social Psychological Foundations of Health and Illness; 2003; 82-106.

15. Fisher W, Fisher JD, Amico K, \& Harman J. An Information-Motivation-Behavioral Skills Model of Adherence to Antiretroviral Therapy. Health Psychology; 2006; 4; 462-473.

16. Hoopes A, Benson S, Howard H, Morrison D, Ko L, \& Shafii T. Adolescent Perspectives on PatientProvider Sexual Health Communication: A Qualitative Study. Journal of Primary Care \& Community Health; 2017; 1-6.

17. Rounds K, McGrath B, \& Walsh E. Perspectives on provider behaviors: A qualitative study of sexual and gender minorities regarding quality of care. Contemporary Nurse; 2013; 44; 99-110.

18. Malta M, Todd C, Stibich M, Garcia T, Pacheco D, \& Bastos F. Patient-provider communication and reproductive health among HIV-positive women in Rio de Janeiro, Brazil. Patient Education and Counseling; 2010; 81; 476-482. 
19. Mimiaga M, Goldhammer H, Belanoff C, Tetu A, \& Mayer K. Men Who Have Sex with Men: Perceptions About Sexual Risk, HIV and Sexually Transmitted Disease Testing, and Provider Communication. Sexually Transmitted Diseases; 2007; 34; 113-119.

20. Fuzzell L, Fedesco H, Alexander S, Fortenberry J, \& Shields C. "I just think that doctors need to ask more questions": Sexual minority and majority adolescents' experiences talking about sexuality with healthcare providers. Patient Education and Counseling; 2016; 99; 1467-1472.

21. De Vasconcelos S, Toskin I, Cooper B, Chollier M, Stephenson R, Blondeel K, Troussier T, Kiarie J. Behaviour change techniques in brief interventions to prevent HIV, STI and unintended pregnancies: A systematic review. PLOS ONE; 2018; 1-27.

22. Stephenson R, Metheny N, Goldenberg T, Bakunina N, De Vasconcelos S, Blondeel K, Kiarie J, Toskin I. Brief Intervention to Prevent Sexually Transmitted Infections and Unintended Pregnancies: Protocol of a Mixed Methods Feasibility Study. JMIR RESEARCH PROTOCOLS, 2020; 9(3); 1-10.

23. Centro Nacional de Epidemiología, Prevención y Control de Enfermedades. Situación epidemiológica del VIH-SIDA en el Perú. Lima: Ministerio de Salud; 2020.

24. Cáceres CF. HIV among homosexual and other men who have sex with men in Latin America and the Caribbean: a hidden epidemic? AIDS. 2002; 16; 23-33.

25. UNAIDS. Global Report: UNAIDS Report on the Global AIDS Epidemic 2013. 2013.

26. Alarcón JO, Pun M, Gutiérrez C, Whittembury A, Tejada R, Suarez L, et al. Estimation and analysis of the incidence of HIV in the adult population of Peru: results of the application of the mathematical model MoT. Rev Peru Med Exp Public Health. 2012; 29; 452-60.

27. Sánchez J, Lama JR, Kusunoki L, Manrique H, Goicochea P, Lucchetti A, et al. HIV-1, sexually transmitted infections, and sexual behavior trends among men who have sex with men in Lima, Peru. J Acquir Immune Defic Syndr. 2007; 44 (5): 578-85.

28. Silva-Santisteban A, Raymond HF, Salazar X, Villayzan J, Leon S, McFarland W, et al. Understanding the HIV/AIDS Epidemic in Transgender Women in Lima, Peru: Results of a Seroepidemiological Study Using a Respondent-Led Sampling. AIDS Behav. 2012; 16; 872-81.

29. National Institute of Statistics and Informatics. Demographic and Family Health Survey-ENDES 2017. Lima: INEl; 2019.

30. Roundtable for Consensus Building in Action against Poverty. Prevención del Embarazo en adolescentes en el actual contexto de Emergencia por COVID-19: Un Problema de Salud Pública, Desarrollo, Desigualdad y Derechos Humanos, Lima; 2020.

31. Ministry of Health. Multisectoral Plan for the Prevention of Pregnancy in Adolescents, 2013-2021. Supreme Decree No. 012-2013-SA.

32. General Direction of strategic interventions in Public Health. Technical Health Standard for Comprehensive adolescent healthcare. Ministry of Health; 2019.

33. Sexual and Reproductive Health Directorate. Norma Técnica de Salud de Planificación Familiar. Lima: Ministerio de Salud; 2017. 